\title{
ON THE STABILITY OF THE FIRST ORDER LINEAR RECURRENCE IN TOPOLOGICAL VECTOR SPACES
}

\author{
MOHAMMAD SAL MOSLEHIAN ${ }^{1}$ AND DORIAN POPA ${ }^{2}$
}

\begin{abstract}
Suppose that $\mathcal{X}$ is a sequentially complete Hausdorff locally convex space over a scalar field $\mathbb{K}, V$ is a bounded subset of $\mathcal{X},\left(a_{n}\right)_{n \geq 0}$ is a sequence in $\mathbb{K} \backslash\{0\}$ with the property $\liminf _{n \rightarrow \infty}\left|a_{n}\right|>1$ and $\left(b_{n}\right)_{n \geq 0}$ is a sequence in $\mathcal{X}$. We show that for every sequence $\left(x_{n}\right)_{n \geq 0}$ in $\mathcal{X}$ satisfying

$$
x_{n+1}-a_{n} x_{n}-b_{n} \in V \quad(n \geq 0)
$$
\end{abstract}

there exists a unique sequence $\left(y_{n}\right)_{n \geq 0}$ satisfying the recurrence $y_{n+1}=$ $a_{n} y_{n}+b_{n}(n \geq 0)$ and for every $q$ with $1<q<\liminf _{n \rightarrow \infty}\left|a_{n}\right|$, there exists $n_{0} \in \mathbb{N}$ such that

$$
x_{n}-y_{n} \in \frac{1}{q-1} \overline{\operatorname{conv}\left(V^{b}\right)} \quad\left(n \geq n_{0}\right) .
$$

\section{INTRODUCTION}

The stability problem of functional equations was originally raised by Ulam [19] in 1940 on a talk at Wisconsin University. The problem posed by Ulam was the following: "Under what conditions does there exist an additive mapping near an approximately additive mapping?" The first answer to the question was given by Hyers in the case of Banach spaces [7]. After Hyers' result many papers dedicated to this topic extending Ulam's problem to other functional equations and generalizing Hyers' result in various directions were published (see e.g. $[4,6,8,9,10,11,16])$. As mentioned in [1] there are much less results on the stability for functional equations in single variable than in several variables. A particular case of equation in single variable is the linear recurrence (difference equation)

$$
x_{n+1}=a_{n} x_{n}+b_{n} .
$$

2010 Mathematics Subject Classification. Primary 39B82; secondary 39A10; 39B72.

Key words and phrases. stability; first order linear recurrence; topological vector spaces; convex hull; balanced hull. 
The results on stability of recurrences play an important role in the theory of dynamical systems and computer science in connection to the notions of shadowing and controlled chaos (see e.g. [12, 13, 18]). The first result on Hyers-Ulam stability of the linear recurrence (1.1) was given by Popa [14] in the case of Banach spaces as follows.

Theorem 1.1. [14] Let $\mathcal{X}$ be a Banach space over the field $\mathbb{K}(\mathbb{R}$ or $\mathbb{C}),\left(\varepsilon_{n}\right)_{n \geq 0}$ a sequence of positive numbers, $\left(a_{n}\right)_{n \geq 0}$ a sequence in $\mathbb{K} \backslash\{0\}$ with the property

$$
\limsup _{n \rightarrow \infty} \frac{\varepsilon_{n}}{\varepsilon_{n-1}\left|a_{n}\right|}<1 \quad \text { or } \quad \liminf _{n \rightarrow \infty} \frac{\varepsilon_{n}}{\varepsilon_{n-1}\left|a_{n}\right|}>1
$$

and $\left(b_{n}\right)_{n \geq 0}$ a sequence in $\mathcal{X}$.

Then there exists a constant $L>0$ such that for every sequence $\left(x_{n}\right)_{n \geq 0}$ in $\mathcal{X}$ satisfying the relation

$$
\left\|x_{n+1}-a_{n} x_{n}-b_{n}\right\| \leq \varepsilon_{n} \quad(n \geq 0)
$$

there exists a sequence $\left(y_{n}\right)_{n \geq 0}$ given by the linear recurrence

$$
y_{n+1}=a_{n} y_{n}+b_{n} \quad(n \geq 0)
$$

with the property

$$
\left\|x_{n}-y_{n}\right\| \leq L \varepsilon_{n-1} .
$$

for some $n_{0} \geq 0$ and all $n \geq n_{0}$.

The above result was extended later by Brzdek, Popa and Xu to the linear recurrences of higher order with constant coefficients [15] and to nonlinear recurrences [2] (see also [3]).

The goal of this paper is to extend Theorem 1.1 to the stability of the linear recurrence (1.1) in topological vector spaces (see also [5]).

If $\mathcal{X}$ is a topological vector space over the field $\mathbb{K},\left(a_{n}\right)_{n \geq 0}$ a sequence in $\mathbb{K}$, $\left(b_{n}\right)_{n \geq 0}$ a sequence in $\mathcal{X}$ and $V$ a bounded subset of $\mathcal{X}$, then the recurrence (1.1) is said to be stable in the spirit of Hyers-Ulam whenever there exists a bounded subset $W$ of $\mathcal{X}$ such that for any sequence $\left(x_{n}\right)_{n \geq 0}$ in $\mathcal{X}$ satisfying

$$
x_{n+1}-a_{n} x_{n}-b_{n} \in V \quad(n \geq 0)
$$

there exists a sequence $\left(y_{n}\right)_{n \geq 0}$ satisfying the linear recurrence

$$
y_{n+1}=a_{n} y_{n}+b_{n}, \quad(n \geq 0)
$$


and

$$
x_{n}-y_{n} \in W
$$

for some $n_{0} \geq 0$ and all $n \geq n_{0}$. For a subset $A$ of a topological vector space $\mathcal{X}$ over the field $\mathbb{K}$ we denote the closedness, the balanced hall and the convex hull of $A$ by $\bar{A}, A^{b}$ and $\operatorname{conv} A$, respectively. Recall that for $\lambda, \mu \in \mathbb{K}$ the following relation holds $(\lambda+\mu) A \subseteq \lambda A+\mu A$. If $\mathbb{K}$ is one of the fields $\mathbb{R}$ or $\mathbb{C}$, $A$ is a convex set and $\lambda, \mu$ are positive numbers, then $(\lambda+\mu) A=\lambda A+\mu A$. The reader is referred to [17] for undefined notation and terminology.

\section{MAin RESULTS}

We give, for the beginning, two auxiliary lemmas which will be used to obtain the main result of this paper. Throughout this section $\mathbb{N}_{0}, \mathbb{R}, \mathbb{C}$ stand as usual for the set of all nonnegative integers, real numbers and complex numbers, respectively. By $\mathbb{K}$ we denote one of the fields $\mathbb{R}$ or $\mathbb{C}$.

Lemma 2.1. Let $\mathcal{X}$ be a sequentially complete Hausdorff locally convex space over $\mathbb{K}$. Suppose that $\left(a_{n}\right)_{n \geq 0}$ is a sequence in $\mathbb{K} \backslash\{0\}$ such that the series $\sum_{n=0}^{\infty} a_{n}$ is absolutely convergent and let $\left(v_{n}\right)_{n \geq 0}$ be a bounded sequence in $\mathcal{X}$. Then the series

$$
\sum_{n=0}^{\infty} a_{n} v_{n}
$$

is convergent in $\mathcal{X}$.

Proof. Let $V$ be an arbitrary neighborhood of 0 and set

$$
\sigma_{n}=\sum_{k=0}^{n} a_{k} v_{k}, \quad s_{n}=\sum_{k=0}^{n}\left|a_{k}\right| \quad(n \geq 0) .
$$

We have to prove that there exists $n_{V} \in \mathbb{N}_{0}$ such that

$$
\sigma_{n+p}-\sigma_{n} \in V
$$

for every $n \geq n_{V}$ and every $p \in \mathbb{N}_{0}$.

Let $U$ be a convex and balanced neighborhood of 0 such that $U \subseteq V$.

Since $A=\left\{v_{n} \mid n \in \mathbb{N}\right\}$ is a bounded set, it follows that there exists $\alpha>0$ such that $A \subseteq \alpha U$. 
From the convergence of $\sum_{n=0}^{\infty}\left|a_{n}\right|$ follows that there exists $n_{0} \in \mathbb{N}$ such that

$$
s_{n+p}-s_{n}<\frac{1}{\alpha} \quad\left(n \geq n_{0}, p \in \mathbb{N}\right) .
$$

We have

$$
\begin{aligned}
\sigma_{n+p}-\sigma_{n} & =\sum_{k=n+1}^{n+p} a_{k} v_{k} \\
& =\left(s_{n+p}-s_{n}\right) \sum_{k=n+1}^{n+p} \frac{a_{k}}{s_{n+p}-s_{n}} \cdot v_{k} \\
& \in \alpha\left(s_{n+p}-s_{n}\right) \sum_{k=n+1}^{n+p} \frac{a_{k}}{s_{n+p}-s_{n}} U .
\end{aligned}
$$

Since $U$ is a balanced set the following relation holds

$$
\frac{a_{k}}{s_{n+p}-s_{n}} U=\frac{\left|a_{k}\right|}{s_{n+p}-s_{n}} U \quad(n+1 \leq k \leq n+p)
$$

hence

$$
\sigma_{n+p}-\sigma_{n} \in \alpha\left(s_{n+p}-s_{n}\right) \sum_{k=n+1}^{n+p} \frac{\left|a_{k}\right|}{s_{n+p}-s_{n}} U .
$$

The convexity of $U$ leads to

$$
\sum_{k=n+1}^{n+p} \frac{\left|a_{k}\right|}{s_{n+p}-s_{n}} U=\left(\sum_{k=n+1}^{n+p} \frac{\left|a_{k}\right|}{s_{n+p}-s_{n}}\right) U=U
$$

therefore

$$
\sigma_{n+p}-\sigma_{n} \in \underbrace{\alpha\left(s_{n+p}-s_{n}\right)}_{<1} U \subseteq U \subseteq V
$$

for every $n \geq n_{0}$ and every $p \in \mathbb{N}$.

Lemma 2.2. Let $\mathcal{X}$ be a vector space over $\mathbb{K},\left(a_{n}\right)_{n \geq 0}$ be a sequence in $\mathbb{K}$, $\left(b_{n}\right)_{n \geq 0}$ a sequence in $\mathcal{X}$ and $\left(x_{n}\right)_{n \geq 0}$ a sequence in $\mathcal{X}$ satisfying the recurrence (1.1). Then

$$
x_{n}=a_{0} a_{1} \ldots a_{n-1} x_{0}+\sum_{k=1}^{n-1} a_{k} \ldots a_{n-1} b_{k-1}+b_{n-1} \quad(n \geq 2) .
$$

Proof. Induction on $n$.

The first stability result for recurrence (1.1) is given in the next theorem. 
Theorem 2.3. Suppose that $\mathcal{X}$ is a sequentially complete Hausdorff locally convex space over $\mathbb{K}, V$ is a bounded subset of $\mathcal{X},\left(a_{n}\right)_{n \geq 0}$ is a sequence in $\mathbb{K} \backslash\{0\}$ with the property $\liminf _{n \rightarrow \infty}\left|a_{n}\right|>1$ and $\left(b_{n}\right)_{n \geq 0}$ is a sequence in $\mathcal{X}$. Then for every sequence $\left(x_{n}\right)_{n \geq 0}$ in $\mathcal{X}$ satisfying

$$
x_{n+1}-a_{n} x_{n}-b_{n} \in V \quad(n \geq 0)
$$

there exists a unique sequence $\left(y_{n}\right)_{n \geq 0}$ satisfying the recurrence

$$
y_{n+1}=a_{n} y_{n}+b_{n} \quad(n \geq 0)
$$

and for every $q$ with $1<q<\liminf _{n \rightarrow \infty}\left|a_{n}\right|$, there exists $n_{0} \in \mathbb{N}$ such that

$$
x_{n}-y_{n} \in \frac{1}{q-1} \overline{\operatorname{conv}\left(V^{b}\right)} \quad\left(n \geq n_{0}\right) .
$$

Proof. Existence. Let $\left(x_{n}\right)_{n \geq 0}$ be a sequence in $\mathcal{X}$ satisfying (2.1) and define the sequence $\left(c_{n}\right)_{n \geq 0}$ by

$$
c_{n}=x_{n+1}-a_{n} x_{n}-b_{n} \quad(n \geq 0) .
$$

Taking account of

$$
\limsup _{n \rightarrow \infty} \frac{\frac{1}{\left|a_{0} \ldots a_{n+1}\right|}}{\frac{1}{\left|a_{0} \ldots a_{n}\right|}}=\limsup _{n \rightarrow \infty} \frac{1}{\left|a_{n+1}\right|}<1
$$

it follows that the series

$$
\sum_{n=0}^{\infty} \frac{1}{\left|a_{0} \ldots a_{n}\right|}
$$

is convergent in view of D'Alembert ratio test.

The boundedness of $\left(c_{n}\right)_{n \geq 0}$ and the convergence of the series (2.5) implies the convergence of the series $\sum_{n=0}^{\infty} \frac{c_{n}}{a_{0} a_{1} \ldots a_{n}}$ in view of Lemma 2.1. Put

$$
\sum_{n=0}^{\infty} \frac{c_{n}}{a_{0} a_{1} \ldots a_{n}}=s \quad(s \in \mathcal{X})
$$

and define the sequence $\left(y_{n}\right)_{n \geq 0}$, by the recurrence (2.2) with

$$
y_{0}=x_{0}+s .
$$


It follows from Lemma 2.2 that

$$
\begin{aligned}
x_{n}-y_{n} & =-\prod_{k=0}^{n-1} a_{k} s+\sum_{k=1}^{n-1} a_{k} \ldots a_{n-1} c_{k-1}+c_{n-1} \\
& =a_{0} a_{1} \ldots a_{n-1}\left(-s+\sum_{k=1}^{n} \frac{c_{k-1}}{a_{0} \ldots a_{k-1}}\right) \\
& =a_{0} \ldots a_{n-1} \sum_{k=0}^{\infty} \frac{c_{n+k}}{a_{0} a_{1} \ldots a_{n+k}} \\
& =\sum_{k=0}^{\infty} \frac{c_{n+k}}{a_{n} a_{n+1} \ldots a_{n+k}} .
\end{aligned}
$$

Let $q$ be a real number such that

$$
1<q<\liminf _{n \rightarrow \infty}\left|a_{n}\right|
$$

Then, there exists $n_{0} \in \mathbb{N}$ such that $\left|a_{n}\right| \geq q$ for every $n \geq n_{0}$.

The following relations hold

$$
\begin{aligned}
\frac{c_{n+k}}{a_{n} \ldots a_{n+k}} & \in \frac{1}{a_{n} \ldots a_{n+k}} V \subseteq \frac{1}{a_{n} \ldots a_{n+k}} V^{b}=\frac{1}{\left|a_{n} \ldots a_{n+k}\right|} V^{b} \\
& \subseteq \frac{1}{q^{k+1}} V^{b} \subseteq \frac{1}{q^{k+1}} \operatorname{conv}\left(V^{b}\right) \quad\left(n, k \in \mathbb{N}_{0}, n \geq n_{0}\right) .
\end{aligned}
$$

From (2.6) and (2.7) we have

$$
\begin{aligned}
\sum_{k=0}^{p} \frac{c_{n+k}}{a_{n} \ldots a_{n+k}} & \in \sum_{k=0}^{p} \frac{1}{q^{k+1}} \operatorname{conv}\left(V^{b}\right) \\
& =\left(\sum_{k=0}^{p} \frac{1}{q^{k+1}}\right) \operatorname{conv}\left(V^{b}\right) \quad\left(n \geq n_{0}\right) .
\end{aligned}
$$

By letting $p \rightarrow \infty$ in (2.8) we get

$$
x_{n}-y_{n} \in \frac{1}{q-1} \overline{\operatorname{conv}\left(V^{b}\right)} \quad\left(n \geq n_{0}\right) .
$$

The existence is proved.

Uniqueness. Let $\left(x_{n}\right)_{n \geq 0}$ be a sequence satisfying (2.1) and suppose that there exists a sequence $\left(y_{n}\right)_{n \geq 0}$ satisfying (2.2) and (2.3) with $y_{0} \neq x_{0}+s$. We 
have

$$
\begin{aligned}
x_{n}-y_{n} & =\prod_{k=0}^{n-1} a_{k}\left(x_{0}-y_{0}\right)+\sum_{k=1}^{n-1} a_{k} \ldots a_{n-1} c_{k-1}+c_{n-1} \\
& =\prod_{k=0}^{n-1} a_{k}\left(x_{0}-y_{0}+\sum_{k=1}^{n} \frac{c_{k-1}}{a_{0} a_{1} \ldots a_{k-1}}\right) .
\end{aligned}
$$

Since

$$
\lim _{n \rightarrow \infty}\left(x_{0}-y_{0}+\sum_{k=1}^{n} \frac{c_{k-1}}{a_{0} a_{1} \ldots a_{k-1}}\right)=x_{0}-y_{0}+s \neq 0
$$

and

$$
\lim _{n \rightarrow \infty}\left|\prod_{k=0}^{n-1} a_{k}\right|=\infty
$$

in view of the convergence of $\sum_{n=0}^{\infty} \frac{1}{\left|a_{0} \ldots a_{n}\right|}$, it follows that $\left(x_{n}-y_{n}\right)_{n \geq 0}$ is an unbounded sequence, a contradiction to (2.3).

A similar result holds for the linear recurrence with constant coefficients.

Theorem 2.4. Let $\mathcal{X}$ be a sequentially complete Hausdorff locally convex space over $\mathbb{K}, V$ a bounded subset of $\mathcal{X}, a \in \mathbb{K},|a|>1$, and $\left(b_{n}\right)_{n \geq 0}$ a sequence in $\mathcal{X}$. Then for every sequence $\left(x_{n}\right)_{n \geq 0}$ in $\mathcal{X}$ satisfying

$$
x_{n+1}-a x_{n}-b_{n} \in V \quad(n \geq 0)
$$

there exists a unique sequence $\left(y_{n}\right)_{n \geq 0}$ fulfilling the recurrence

$$
y_{n+1}=a y_{n}+b_{n} \quad(n \geq 0)
$$

such that

$$
x_{n}-y_{n} \in \frac{1}{|a|-1} \cdot \overline{\operatorname{conv}\left(V^{b}\right)} \quad(n \geq 0) .
$$

Proof. Denoting $c_{n}:=x_{n+1}-a x_{n}-b_{n}(n \geq 0)$ it follows that the series $\sum_{n=0}^{\infty} \frac{c_{n}}{a^{n+1}}$ is convergent in view of Lemma 2.1. Put

$$
\sum_{n=0}^{\infty} \frac{c_{n}}{a^{n+1}}=s \quad(s \in \mathcal{X})
$$


and define $\left(y_{n}\right)_{n \geq 0}$ by the recurrence (2.10) with $y_{0}=x_{0}+s$. It follows, as in the proof of Theorem 2.3, that

$$
x_{n}-y_{n}=\sum_{k=0}^{\infty} \frac{c_{n+k}}{a^{k+1}} \quad(n \geq 0)
$$

and

$$
\frac{c_{n+k}}{a^{k+1}} \in \frac{1}{|a|^{k+1}} \cdot V^{b} \subseteq \frac{1}{|a|^{k+1}} \cdot \operatorname{conv}\left(V^{b}\right) .
$$

Then

$$
\sum_{k=0}^{p} \frac{c_{n+k}}{a^{k+1}} \in\left(\sum_{k=0}^{p} \frac{1}{|a|^{k+1}}\right) \operatorname{conv}\left(V^{b}\right) .
$$

Now by letting $p \rightarrow \infty$ in (2.11) we get (2.10).

The uniqueness follows analogously to Theorem 2.3.

Corollary 2.5. Suppose that $\mathcal{X}$ is a Banach space over $\mathbb{K}, \varepsilon>0,|a|>1$ and $\left(b_{n}\right)_{n \geq 0}$ is a sequence in $\mathcal{X}$. Then for every sequence $\left(x_{n}\right)_{n \geq 0}$ in $\mathcal{X}$ satisfying

$$
\left\|x_{n+1}-a x_{n}-b_{n}\right\| \leq \varepsilon \quad(n \geq 0)
$$

there exists a unique sequence $\left(y_{n}\right)_{n \geq 0}$ satisfying the recurrence

$$
y_{n+1}=a y_{n}+b_{n} \quad(n \geq 0)
$$

such that

$$
\left\|x_{n}-y_{n}\right\| \leq \frac{\varepsilon}{|a|-1} \quad(n \geq 0)
$$

Proof. Use Theorem 2.4 with $a_{n}=a$ and take $V$ to be the closed ball of center 0 with radius $\varepsilon$.

Remark 2.6. If $\liminf _{n \rightarrow \infty}\left|a_{n}\right| \leq 1$, then the conclusion of Theorem 2.3 may be not true.

To see this, set $\mathcal{X}=\mathbb{K}=\mathbb{R}$, take $1 \leq r<2$, let $V$ be the interval $(-r, r)$, $a_{n}=r, b_{n}=0(n \geq 0)$ and consider the sequence $\left(x_{n}\right)_{n \geq 0}$ given by $x_{n+1}-r x_{n}=$ $1(n \geq 0), x_{0}=0$. Then $x_{n}=\sum_{j=1}^{n-1} r^{j}$. One can observe that for any sequence $\left(y_{n}\right)_{n \geq 0}$ satisfying the recurrence $y_{n+1}=r y_{n}$ we have

$$
\sup _{n \rightarrow \infty}\left|x_{n}-y_{n}\right|=\infty .
$$


In fact,

$$
\sup _{n \rightarrow \infty}\left|x_{n}-y_{n}\right|=\sup \left(\left\{\left|\sum_{j=0}^{n-1} r^{j}-r^{n} y_{0}\right|: n=1,2, \cdots\right\} \cup\left\{\left|y_{0}\right|\right\}\right) .
$$

If $y_{0} \leq 0$, then $\sup _{n \rightarrow \infty}\left|x_{n}-y_{n}\right|=\infty$. Let us assume $y_{0}>0$. There exist positive integers $k_{0}$ and $n_{0}$ such that $r^{k_{0}} \geq y_{0}$ and $\sum_{j=0}^{n-1} r^{j} \geq r^{n}$ for all $n \geq n_{0}$. Then

$$
\sum_{j=k_{0}}^{n-1+k_{0}} r^{j}-r^{n} y_{0} \leq r^{k_{0}}\left(\sum_{j=0}^{n-1} r^{j}-r^{n}\right)=(2-r) r^{n+k_{0}}-r^{k_{0}}
$$

for all $n \geq \max \left\{k_{0}, n_{0}\right\}$. Therefore $\left|\sum_{j=0}^{n-1} r^{j}-r^{n} y_{0}\right| \rightarrow \infty$ as $n \rightarrow \infty$. Hence $\sup _{n \rightarrow \infty}\left|x_{n}-y_{n}\right|=\infty$.

Theorem 2.7. Let $\mathcal{X}$ be a Hausdorff topological vector space over the field $\mathbb{K}$, $V$ a bounded subset of $\mathcal{X},\left(a_{n}\right)_{n \geq 0}$ a sequence in $\mathbb{K}$ with $\limsup _{n \rightarrow \infty}\left|a_{n}\right|<1$ and $\left(b_{n}\right)_{n \geq 0}$ a sequence in $\mathcal{X}$. Then there exists a positive number $M$ such that for every sequence $\left(x_{n}\right)_{n \geq 0}$ in $\mathcal{X}$ satisfying

$$
x_{n+1}-a_{n} x_{n}-b_{n} \in V \quad(n \geq 0)
$$

there exists a sequence $\left(y_{n}\right)_{n \geq 0}$ satisfying the linear recurrence

$$
y_{n+1}=a_{n} y_{n}+b_{n} \quad(n \geq 0)
$$

such that

$$
x_{n}-y_{n} \in M \cdot \operatorname{conv}\left(V^{b}\right) \quad\left(n \geq n_{0}\right) .
$$

Proof. Let $\left(c_{n}\right)_{n \geq 0}$ be defined by (2.4), as in the proof of Theorem 2.3 and $\left(y_{n}\right)_{n \geq 0}$ be given by the recurrence (2.12) with $y_{0}=x_{0}$. Then

$$
x_{n}-y_{n}=\sum_{k=1}^{n-1} a_{k} \ldots a_{n-1} c_{k-1}+c_{n-1} \quad(n \geq 2) .
$$

Choose $q \in \mathbb{R}$ such that

$$
\limsup _{n \rightarrow \infty}\left|a_{n}\right|<q<1 .
$$

Then there exists $n_{0} \in \mathbb{N}$ such that

$$
\left|a_{n}\right| \leq q \quad\left(n \geq n_{0}\right) .
$$


For $k \in \mathbb{N}, k \geq n_{0}$ we have

$$
\left|a_{k} \ldots a_{n-1}\right| \leq q^{n-k}
$$

and for $k<n_{0}$

$$
\begin{aligned}
\left|a_{k} \ldots a_{n-1}\right| & =\left|a_{k} \ldots a_{n_{0}-1}\right| \cdot\left|a_{n_{0}} \ldots a_{n-1}\right| \\
& \leq q^{n-n_{0}}\left|a_{k} \ldots a_{n_{0}-1}\right| \\
& \leq\left|a_{k} \ldots a_{n_{0}-1}\right| .
\end{aligned}
$$

Then, the same argument as in the proof of Theorem 2.3 follows

$$
\begin{aligned}
x_{n}-y_{n} & =\sum_{k=1}^{n_{0}-1} a_{k} \ldots a_{n-1} c_{k-1}+\sum_{k=n_{0}}^{n-1} a_{k} \ldots a_{n-1} c_{k-1}+c_{n-1} \\
& \in \sum_{k=1}^{n_{0}-1}\left|a_{k} \ldots a_{n_{0}-1}\right| \cdot V^{b}+\sum_{k=n_{0}}^{n-1} q^{n-k} \cdot V^{b}+V^{b} \\
& \subseteq \sum_{k=1}^{n_{0}-1}\left|a_{k} \ldots a_{n_{0}-1}\right| \operatorname{conv}\left(V^{b}\right)+\frac{q}{1-q} \operatorname{conv}\left(V^{b}\right)+\operatorname{conv}\left(V^{b}\right) \\
& =\left(\sum_{k=1}^{n_{0}-1}\left|a_{k} \ldots a_{n_{0}-1}\right|+\frac{1}{1-q}\right) \operatorname{conv}\left(V^{b}\right) .
\end{aligned}
$$

Choosing

$$
M:=\sum_{k=1}^{n_{0}-1}\left|a_{k} \ldots a_{n_{0}-1}\right|+\frac{1}{1-q} .
$$

In Theorem 2.7 the sequence $\left(y_{n}\right)_{n \geq 0}$ is not necessary uniquely determined (see [14, Remark 2.2]).

Theorem 2.8. Let $\mathcal{X}$ be a Hausdorff topological vector space over the field $\mathbb{K}$, $V$ a bounded subset of $\mathcal{X}, a \in \mathbb{K},|a|<1$, and $\left(b_{n}\right)_{n \geq 0}$ a sequence in $\mathcal{X}$. Then for every sequence $\left(x_{n}\right)_{n \geq 0}$ in $\mathcal{X}$ satisfying (2.9) there exists a sequence $\left(y_{n}\right)_{n \geq 0}$ in $\mathcal{X}$ fulfilling the recurrence (2.10) such that

$$
x_{n}-y_{n} \in \frac{1}{1-|a|} \cdot \operatorname{conv}\left(V^{b}\right) \quad(n \geq 0) .
$$


Proof. Let $c_{n}:=x_{n+1}-a x_{n}-b_{n}(n \geq 0)$ and $\left(y_{n}\right)_{n \geq 0}$ be given by the recurrence (2.10) with $y_{0}=x_{0}$. Then

$$
x_{n}-y_{n}=\sum_{k=1}^{n} a^{n-k} c_{k-1} \quad(n \geq 1) .
$$

It follows that

$$
\begin{aligned}
x_{n}-y_{n} & \in \sum_{k=1}^{n} a^{n-k} V \subseteq \sum_{k=1}^{n}|a|^{n-k} V^{b} \\
& \subseteq \sum_{k=1}^{n}|a|^{n-k} \operatorname{conv}\left(V^{b}\right) \\
& =\left(\sum_{k=1}^{n}|a|^{n-k}\right) \operatorname{conv}\left(V^{b}\right) \\
& =\frac{1-|a|^{n}}{1-|a|} \operatorname{conv}\left(V^{b}\right) \\
& \subseteq \frac{1}{1-|a|} \operatorname{conv}\left(V^{b}\right) \quad(n \geq 0) .
\end{aligned}
$$

Corollary 2.9. Suppose that $\mathcal{X}$ is a normed space over $\mathbb{K}, \varepsilon>0,|a|<1$ and $\left(b_{n}\right)_{n \geq 0}$ is a sequence in $\mathcal{X}$. Then for every sequence $\left(x_{n}\right)_{n \geq 0}$ in $\mathcal{X}$ satisfying

$$
\left\|x_{n+1}-a x_{n}-b_{n}\right\| \leq \varepsilon \quad(n \geq 0)
$$

there exist a positive number $M$ and a sequence $\left(y_{n}\right)_{n \geq 0}$ satisfying the recurrence

$$
y_{n+1}=a y_{n}+b_{n} \quad(n \geq 0)
$$

such that

$$
\left\|x_{n}-y_{n}\right\| \leq \frac{1}{1-|a|} \varepsilon \quad(n \geq 0) .
$$

Proof. Use Theorem 2.8 with $a_{n}=a$ and take $V$ to be the closed ball of center 0 with radius $\varepsilon$.

\section{REFERENCES}

[1] R.P. Agarwal, B. Xu and W. Zhang, Stability of functional equations in single variable, J. Math. Anal. Appl. 288 (2003), 852-869. 
[2] J. Brzdek, D. Popa and B. Xu, The Hyers-Ulam stability of the nonlinear recurrences, J. Math. Anal. Appl. 335 (2007), 443-449.

[3] J. Brzdek, D. Popa and B. Xu, Note on the nonstability of the linear recurrence, Abh. Math. Sem. Univ. Hamburg 76 (2006), 183-189.

[4] S. Czerwik, Functional Equations and Inequalities in Several Variables, World Scientific, New Jersey, London, Singapore, Hong Kong, 2002.

[5] S. Czerwik and M. Adam, On the stability of the quadratic functional equation in topological spaces, Banach J. Math. Anal. 1 (2007), no. 2, 245-251.

[6] G.L. Forti, Hyers-Ulam stability of functional equations in several variables, Aequationes Math. 50 (1995), 143-190.

[7] D.H. Hyers, On the stability of the linear functional equation, Proc. Nat. Acad. Sci. USA 27 (1941), 222-224.

[8] D.H. Hyers, G. Isac and Th.M. Rassias, Stability of Functional Equations in Several Variables, Birkhäuser, Boston, 1998.

[9] S.-M. Jung, Hyers-Ulam-Rassias Stability of Functional Equations in Mathematical Analysis, Hadronic Press, Palm Harbor, Florida, 2001.

[10] M. Kuczma, Functional Equations in Single Variable, Polish Scientific Publishers, Warszawa, 1968.

[11] M.S. Moslehian, Ternary derivations, stability and physical aspects, Acta Applicandae Math. 100 (2008), no. 2, 187-199.

[12] K. Palmer, Shadowing in Dynamical Systems, Kluwer Academic Press, 2000.

[13] S. Pilyugin, Shadowing in Dynamical Systems, Lecture Notes in Mathematics 1706 , Springer-Verlag, 1999.

[14] D. Popa, Hyers-Ulam-Rassias stability of a linear recurrence, J. Math. Anal. Appl. 309 (2005), 591-597.

[15] D. Popa, Hyers-Ulam stability of the linear recurrence with constant coefficients, Adv. in Diff. Eq. 2005-2 (2005), 101-107.

[16] Th.M. Rassias(ed.), Functional Equations, Inequalities and Applications, Kluwer Academic Publishers, Dordrecht, Boston and London, 2003.

[17] W. Rudin, Functional analysis, Second edition. International Series in Pure and Applied Mathematics. McGraw-Hill, Inc., New York, 1991.

[18] J. Tabor, General stability of functional equations of linear type, J. Math. Anal. Appl. 1 (2007), 192-200.

[19] S.M. Ulam, Problems in Modern Mathematics, Wiley, New York, 1964.

1 Department of Pure Mathematics, Center of Excellence in Analysis on Algebraic Structures (CEAAS), Ferdowsi University of Mashhad, P.O. Box 1159, MashHad 91775, Iran.

E-mail address: moslehian@ferdowsi.um.ac.ir and moslehian@ams.org

URL: http://profsite.um.ac.ir/ moslehian/ 
2 Technical University, Department of Mathematics, Str. C. Daicoviciu 15, 400020 Cluj-Napoca, Romania.

E-mail address: Popa.Dorian@math.utcluj.ro 